\title{
DOCTORADOS EN EDUCACIÓN DE LA REGIÓN ANDINA: LAS COMPETENCIAS COMO BÁSICOS CURRICULARES
}

Maria Eugenia Navas Ríos

Universidad de Cartagena

Colombia

Emperatriz Londoño Aldana Universidad de Cartagena Colombia 
Panorama Económico, Vol. 27 - No. 1 (Enero - Marzo de 2019), pp. 116-133

María Eugenia Navas Ríos

Emperatriz Londoño Aldana

JEL: A13, A19, A23

\title{
Doctorados en educación de la Región Andina: las competencias como básicos curriculares
}

\begin{abstract}
Resumen
Partiendo del conocimiento de las bondades de armonizar e integrar regiones, países, instituciones y programas, Tomando como referente experiencias europeas caso Tuning; experiencias en el ámbito Iberoamericano como la creación del Espacio Iberoamericano del Conocimiento (EIC) y latinoamericanas como la conformación del Mercosur educativo y el proyecto Tuning-América Latina 2004-2006, éste trabajo se planteó como objetivo identificar las competencias que debe tener un doctor en educación desde las dimensiones del ser, convivir, conocer y hacer en forma tal que responda a las necesidades de la emergente y actual sociedad del conocimiento. Este grupo de competencias se constituye en el corazón de las propuestas de formación doctoral en educación en la región andina como básicos curriculares de las mismas. Para el logro del objetivo se empleó, la técnica Delphi, la cual, permitió hallar el consenso acerca del grupo de competencias. Se emplearon parámetros estadísticos para su verificación y respectiva comprobación. Se realizó un diagnóstico de las competencias que actualmente se promueven desde las 22 propuestas ofertadas en la región por las diferentes universidades de los países miembros de la misma, encontrándose una tendencia a privilegiar las competencias del saber y del hacer; y por último se hace un recorrido por lo que en este tema se ha avanzado en países de la unión europea y los estados unidos, para que sirvan como referentes internacionales concluyendo en la conformación de cinco grupos específicos de competencias a promocionar desde los doctorados en educación que van a servir como elementos de integración y armonización de los países desde dichas propuestas, asegurando además, su pertinencia en el ámbito internacional.
\end{abstract}

Palabras clave: Básicos curriculares, Integración, Competencias, doctorados

\section{Doctorats en éducation de la Région Andine: les compétences comme bases curriculaires}

\section{Résumé}

Prendre en considération la connaissance des avantages de l'harmonisation et de l'intégration des régions, pays, institutions et programmes. Prenant comme référence les expériences européennes Tuning case; des expériences dans le domaine ibéro-américain telles que la création de la zone de connaissance ibéro-américaine (EIC) et des expériences latino-américaines telles que la formation du Mercosur éducatif et le projet Tuning-Amérique latine 2004-2006. L'objectif actuel du travail était d'identifier les compétences qu'un docteur en éducation devrait avoir des dimensions d'être, de vivre, de savoir et de faire d'une manière qui réponde aux besoins de la société du savoir émergente et actuelle. Ce groupe de compétences est au cœur des propositions de formation doctorale en éducation dans la région andine comme cursus de base. Pour atteindre l'objectif, la technique Delphi a été utilisée, ce qui a permis de trouver un consensus sur le groupe de compétences. Des paramètres statistiques ont été utilisés pour la vérification et la vérification respective. Un diagnostic a été fait des compétences qui sont actuellement promues à partir des 22 propositions proposées dans la région par les différentes universités des pays membres de la même, en trouvant une tendance à privilégier les compétences de la connaissance et de l'action. Enfin, une tournée est faite de ce qui a été avancé dans ce domaine dans les pays de l'Union européenne et aux États-Unis, afin qu'ils servent de références internationales, aboutissant à la formation de cinq groupes spécifiques de compétences à promouvoir à partir des doctorats en éducation. qu'elles serviront d'éléments d'intégration et d'harmonisation des pays à partir de ces propositions, garantissant également leur pertinence sur la scène internationale.

Mots clés: Programmes de base, intégration, compétences, doctorats. 
Panorama Económico, Vol. 27 - No. 1 (Enero - Marzo de 2019), pp. 116-133

María Eugenia Navas Ríos

Emperatriz Londoño Aldana

JEL: A13, A19, A23

\title{
Education doctorates in the Andean Region: competences as curricular basics
}

\begin{abstract}
Take into consideration knowledge of the benefits of harmonization and integration of regions, countries, institutions and programs. Taking as reference the European experiences Tuning case; experiences in the Ibero-American field such as the creation of the Ibero-American knowledge zone (EIC) and Latin American experiences such as the training of the educational Mercosur and the Tuning-Latin America project 2004-2006. The current objective of the work was to identify the skills that a doctor in education should have dimensions of being, living, knowing and doing in a way that meets the needs of the emerging and current knowledge society. This group of skills is at the heart of doctoral training proposals in education in the Andean region as a basic course. To achieve the objective, the Delphi technique was used, which made it possible to find a consensus on the group of skills. Statistical parameters were used for the verification and the respective verification. A diagnosis has been made of the skills that are currently promoted from the 22 proposals proposed in the region by the different universities of the member countries of the same, finding a tendency to favor skills of knowledge and action. Finally, a tour is made of what has been advanced in this area in the countries of the European Union and in the United States, so that they serve as international references, leading to the formation of five specific groups of skills to promote from doctorates in education. that they will serve as elements of integration and harmonization of countries based on these proposals, also guaranteeing their relevance on the international scene.
\end{abstract}

Key words: Basic curricula, Integration, Competences, doctorates. 


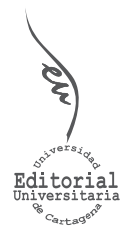

Artículo de Investigación

\section{Doctorados en educación de la Región Andina: las competencias como básicos curriculares}

INFORMACIÓN DEL ARTíCULO

Recepción del artículo: 23/08/2018

Concepto de evaluación: $30 / 09 / 2018$

Aceptación del artículo: 24/11/2018
María Eugenia Navas Ríos*

Universidad de Cartagena, Colombia

Emperatriz Londoño Aldana* Universidad de Cartagena, Colombia

\section{INTRODUCCIÓN}

Estos acuerdos, consensos, puntos comunes, convergencias o básicos curriculares han sido desarrollados por diversos autores a través del tiempo con diferentes connotaciones. Para John Dewey (1916: 125), el núcleo curricular se relaciona con los conocimientos y destrezas que la escuela provee a todos los estudiantes para actuar en la vida democrática, es decir, conocimientos y destrezas que todos por igual deben tener, finalizada su formación escolar. Goodlad y Su (1992) expresan el núcleo del currículum, o core currículum, como la especificación de aquellas áreas que se juzgan como esenciales en la formación de todo estudiante; en este caso, los autores manejan como comunes las áreas. Mac Connell señala que: "el core currículum está constituido por todas las experiencias educacionales que se consideran importantes para cada ciudadano en nuestra democracias" lo común, desde este autor, son la experiencias educativas. Para Smith,(1940: 25), "el core currículum representa la suma total de los problemas que los jóvenes enfrentan y los que consideran de importancia social" (1945: 164); lo común viene dado por las necesidades de los jóvenes, jerarquizadas desde lo social. Por esta misma línea se encuentra Magendzo, quien en su libro Currículum, Educación para la Democracia expresa: "deben ser consideradas como core currículum aquellas experiencias que son fundamentales para todos los educandos porque responden a sus necesidades comunes en el plano individual y social y les hace ciudadanos competentes de una comunidad democrática" (1996: 82).

Dentro de los beneficios de armonizar las propuestas desde las competencias como básicos curriculares, se presentan: una mejor formación de las personas, entendidas como "indispensable o imprescindible" para lograr el éxito de las organizaciones (Passos y Arias, 2016) , mayores conocimientos, mejora de la calidad, movilidad, empleabilidad, promoción de la cooperación interuniversitaria y nuevas figuras del profesorado; a su vez, facilitan la formulación de indicadores de calidad que pueden ser comprendidos y compartidos, facilitando los procesos de evaluación y acreditación de dichos

\footnotetext{
*Docentes de carrera del Programa de Administración de Empresas de la Universidad de Cartagena. Autor de correspondencia: mnavar@unicartagena.edu.co
} 
programas a nivel nacional, regional e internacional. Se enfatiza en que el consenso acerca de las competencias como básico curricular que integra, no limita, al contrario, esos son los comunes pero por encima de estos se pueden adicionar otras competencias que definen o le dan personalidad a cada programa de doctorado en educación en función de su pertinencia frente a las necesidades del contexto donde se desarrollan, es decir, deja espacio para la diversidad, la libertad y la autonomía, consolidando una propuesta curricular dinámica, que está en un proceso constante de evolución en una sociedad siempre cambiante, cuyas necesidades y valores están llamados a servir (Tuning, 2003).

La investigación es de tipo plurimetodológico, con base en la hermenéutica, pues la interpretación, su característica principal, es el proceso que la acompaña de principio a fin. Se recurre, a la metodología comparativa, siguiendo las cuatro fases: Descripción, la interpretación; yuxtaposición o confrontación preliminar de datos de aquí se desprende información sobre semejanzas y diferencias, aparece aquí la figura del criterio de comparación, como el ideal ser que va a permitir la armonización de las propuestas en función de él. El criterio de comparación se construyó utilizando la técnica Delphi, la cual contó con la participación de 17 expertos doctores en educación con experiencia y autonomía en el conocimiento que podían aportar al trabajo, procedentes de los países andinos. Y por último esta la cuarta fase denominada Fase de Comparación se emiten las conclusiones del estudio, dando origen a un nuevo conocimiento que surge de la comparación y que asegura la posibilidad de la armonización, respectando las diferencias desde el consenso en cuanto a las competencias del doctor en educación. (Garcia,2000)

Como estrategias metodológicas se privilegió la encuesta individual estructurada que se aplicó al grupo de expertos, el análisis de documentos con sus cinco etapas (Sandoval,1996) y la técnica Delphi (Dalkey, 1969; Delbecq, Van de Ven y Gustafson, 1975). Esta técnica grupal consiste en un proceso iterativo de cuestionario-resultados-cuestionario. Se prolonga hasta que se logra un determinado objetivo: disponer de información de calidad, como consecuencia de la toma de decisiones en grupo (Linstone y Turoff, 1975).

El estudio necesitó dos tipos de muestra. La primera la constituyeron las propuestas de formación doctoral en educación de cada uno de los países que conforman la comunidad Andina y que en los actuales momentos se encuentran ofertadas 23. La segunda la conformó el grupo de expertos un total de17.

Para medir el grado de consistencia interna (fiabilidad) de la escala empleada en la encuesta, se recurrió al cálculo del Coeficiente Cronbach (Cronbach 1951) y para la validación de lo cualitativo en el análisis realizado en esta investigación, se emplearon las técnicas univarable, bivariables y multivariables (el análisis de grupo). El software empleado en la codificación, tabulación y análisis de resultados fue el DYANE del español Miguel Santesmases

\section{COMPETENCIAS EN EL ESPACIO EUROPEO}

De acuerdo con el proceso de Bolonia y lo acordado en la Reunión de Ministros Europeos responsables de Educación Superior, realizada en Bergen, los días 19 y 20 de mayo de 2005, se hizo un llamado urgente a las universidades a asegurar que sus programas doctorales promuevan la formación interdisciplinar y el desarrollo de competencias transferibles, de acuerdo con las necesidades de un amplio mercado de trabajo. 
Al respecto, los descriptores de Dublín enuncian, al término del tercer ciclo (doctorado), los alumnos demostrarán / podrán demostrar:

1. Poseer y comprender conocimientos que incluyan la comprensión sistemática de un área de estudio y el dominio de las habilidades y métodos de investigación relacionados con dicha área.

2. Aplicación de conocimientos y comprensión a través de la capacidad de concebir, diseñar, poner en práctica y adoptar un proceso sustancial de investigación con seriedad académica.

3. Que haya realizado una contribución a través de una investigación original que amplíe las fronteras del conocimiento desarrollando un corpus sustancial, que merezca una publicación referenciada a escala nacional o internacional.

4.Capacidad de emitir juicios a través del análisis crítico, evaluación y síntesis de ideas nuevas y complejas.

5. Capacidad de comunicarse con sus colegas, con la comunidad académica en su conjunto y con la sociedad en general acerca de sus áreas de conocimiento.

6.Habilidades de aprendizaje que les permitan fomentar, en contextos académicos y profesionales, el avance tecnológico, social o cultural dentro de una sociedad basada en el conocimiento.

Típicamente, los poseedores de tal calificación están en capacidad de: elaborar juicios en situaciones complejas y en campos especializados, a menudo sin contar con datos completos, poder comunicar sus ideas y conclusiones en forma clara y efectiva a especialistas y audiencias no especializadas y continuar emprendiendo investigaciones puras o aplicadas, contribuyendo substancialmente al desarrollo de nuevas técnicas, ideas o acercamientos. Y tendrán las calidades y las habilidades transferibles necesarias para el empleo, que requieren el ejercicio de la responsabilidad y en gran parte autonomía, iniciativa en situaciones complejas e imprevisibles en ambientes profesionales o equivalentes.

\section{ESTADO ACTUAL DE LAS COMPETENCIAS EN LA FORMACIÓN DOCTORAL EN LA REGIÓN ANDINA.}

Desde una mirada latinoamericana, y específicamente desde la región andina, el tipo de doctor que está egresando, en cuanto a la formación de competencias es el siguiente:

En Bolivia se hace mucho énfasis en las competencias del hacer, seguido del conocer, poco en el ser y el convivir. La Universidad Mayor de San Andrés, por ejemplo, en su perfil de egreso, deja bien claro: es un investigador del más alto nivel, capaz de desarrollar tareas de administración educativa, de enseñanza y asesoría; capaz de producir y desarrollar modelos y teorías educativas y tendrá una actitud crítica, flexible y creativa frente a los problemas educativos. Se concluye: la Universidad Mayor de San Andrés, actualmente, está trabajando en la adquisición de las metacompetencias que debe poseer un doctor y que cobijan las demás competencias. 
En Colombia, al igual que en Bolivia y Perú, continúa prevaleciendo en el enunciado de los perfiles de egreso de los respectivos programas doctorales las competencias del hacer, tal es el caso de la Universidad de Antioquia, que en términos muy generales habla de su egresado como un investigador independiente capaz de crear y coordinar grupos de investigación; capaz de producir nuevo conocimiento o ampliar el existente en el campo de la educación y en el énfasis específico, conforme a las tendencias internacionales de las publicaciones e impacto científico (Escobar y Pico, 2015; Vergara et al., 2015). Las dos redes de instituciones que ofertan los otros dos programas doctorales en educación explicitan las competencias del saber y del hacer y además hablan de la interdisciplinariedad y las relaciones interinstistucionales en el hacer del doctor; específicamente RUDECOLOMBIA enuncia su perfil de egresado desde los dominios y competencias, entendiéndose los dominios como las competencias del saber, conocer, y las competencias como las del hacer.

En el Perú continúa el predominio de enfatizar en las competencias del hacer, seguido del saber. El hacer en este país se muestra explícito en el campo de la investigación, la docencia y la asesoría, como lo demuestra el perfil del egresado de la Universidad Nacional de Trujillo.

En Venezuela, al momento de definir el perfil del egresado, combinan el saber con el hacer en el área educativa, y encontramos perfiles muy generales como el enunciado por la Universidad Nacional Experimental Simón Rodríguez.

\section{ANÁLISIS DE COMPETENCIAS PERTINENTES DEL DOCTOR EN EDUCACIÓN DESDE EL GRUPO DE EXPERTOS.}

Para el análisis del grupo de competencias que debe tener un doctor en educación desde la investigación, y con el aval del grupo de expertos consultados y los respectivos estadísticos aplicados, estas se han agrupado teniendo en cuenta lo referido por Jacques Delors (1996) en la Comisión Internacional sobre la Educación para el Siglo XXI, que apuntan a que la educación se estructure sobre la base de cuatro aprendizajes fundamentales: aprender a conocer, aprender a hacer, aprender a convivir y aprender a ser.

Se consideró además que existen unas competencias generales que poseen o deben poseer los doctores para su desempeño efectivo, y son denominadas metacompetencias (Navio 2001), las cuales pueden influir en la adquisición de competencias más específicas. Estas metacompetencias son útiles para hacer frente a situaciones complejas e imprevistas propias de nuestra sociedad.

\subsection{Competencias generales o metacompetencias.}

Se conformaron teniendo en cuenta la mayor frecuencia en las respuestas (en este caso oscilaron entre el 80 y 93\%), la menor desviación estándar (va desde 0,59 a 1,06) y las medias aritméticas más altas (se encontraron en un rango entre 4,25 y 4,68). Sus respectivas cifras se pueden apreciar en la tabla 3.1. 
Tabla 3.1.

Competencias generales - Metacompetencias

\begin{tabular}{|c|c|c|c|c|}
\hline & & $\%$ & DS & MA \\
\hline 27. & Capacidad crítica. & 93 & 0.59 & 4.62 \\
\hline 49. & Actitud crítica y creativa. & 93 & 0.59 & 4.62 \\
\hline 46. & $\begin{array}{l}\text { Rigor metodológico. El rigor implica } \\
\text { humildad, capacidad de autocrítica } \\
\text { y, a la vez, capacidad de crítica } \\
\text { constructiva. }\end{array}$ & 93 & 0.76 & 4.68 \\
\hline 31. & Pensamiento crítico. & 87 & 1.06 & 4.50 \\
\hline 53. & Desarrollar el pensamiento crítico. & 87 & 0.86 & 4.43 \\
\hline 28. & Análisis. & 86 & 0.86 & 4.50 \\
\hline 38. & $\begin{array}{l}\text { Conocer a fondo la } \\
\text { disciplina específica. }\end{array}$ & 85 & 0.75 & 4.25 \\
\hline 29. & Autonomía. & 80 & 0.92 & 4.37 \\
\hline
\end{tabular}

Para verificar la relación entre ellas, recurrimos al análisis de correlación lineal y al nivel de significación (p) y quedó conformada la matriz de coeficientes de correlación simple de las metacompetencias.

Matriz de coeficientes de correlación simple de las metacompetencias.

\begin{tabular}{|c|c|c|c|c|c|c|c|}
\hline COM27 & COM28 & COM29 & COM31 & COM38 & COM46 & COM49 & COM53 \\
\hline COM 271,0000 & 0,7275 & 0,8106 & 0,7008 & 0,3556 & 0,7732 & 0,8039 & 0,3919 \\
\hline$p=0,0000$ & 0,0008 & 0,0001 & 0,0016 & 0,1600 & 0,0002 & 0,0001 & 0,1184 \\
\hline COM 280,7275 & 1,0000 & 0,4739 & 0,5368 & 0,3911 & 0,6219 & 0,5743 & 0,3173 \\
\hline$p=0,0008$ & 0,0000 & 0,0535 & 0,0254 & 0,1193 & 0,0072 & 0,0152 & 0,2134 \\
\hline COM 290,8106 & 0,4739 & 1,0000 & 0,4329 & 0,4091 & 0,5877 & 0,6382 & 0,2715 \\
\hline$p=0,0001$ & 0,0535 & 0,0000 & 0,0814 & 0,1017 & 0,0125 & 0,0055 & 0,2908 \\
\hline COM31 0,7008 & 0,5368 & 0,4329 & 1,0000 & 0,2440 & 0,5497 & 0,7005 & 0,5002 \\
\hline$p=0,0016$ & 0,0254 & 0,0814 & 0,0000 & 0,3444 & 0,0215 & 0,0016 & 0,0398 \\
\hline COM38 0,3556 & 0,3911 & 0,4091 & 0,2400 & 1,0000 & 0,4026 & 0,5653 & 0,6208 \\
\hline$p=0,1600$ & 0,1193 & 0,1017 & 0,3444 & 0,0000 & 0,1078 & 0,0173 & 0,0074 \\
\hline COM46 0,7732 & 0,6219 & 0,5877 & 0,5497 & 0,4026 & 1,0000 & 0,8852 & 0,6137 \\
\hline$p=0,0002$ & 0,0072 & 0,0125 & 0,0215 & 0,1078 & 0,0000 & 0,0000 & 0,0083 \\
\hline COM49 0,8039 & 0,5743 & 0,6382 & 0,7005 & 0,5653 & 0,8852 & 1,0000 & 0,7784 \\
\hline$p=0,0001$ & 0,0152 & 0,0055 & 0,0016 & 0,0173 & 0,0000 & 0,0000 & 0,0002 \\
\hline COM53 0,3919 & 0,3173 & 0,2715 & 0,5002 & 0,6208 & 0,6137 & 0,7784 & 1,0000 \\
\hline $\mathrm{p}=0,1184$ & 0,2134 & 0,2908 & 0,0398 & 0,0074 & 0,0083 & 0,0002 & 0,0000 \\
\hline
\end{tabular}


Teniendo en cuenta que una alta correlación, se da, en la medida en que estas se aproximen a uno (1), en la matriz de correlación simple de las metacompetencias, se observa que en su gran mayoría, se encuentra un coeficiente de correlación por encima del 0,5, a excepción de la variable 38 (Conocer a fondo la disciplina específica), que presenta una baja correlación con la mayoría.

Si se analiza el nivel de significación, que es mayor en la medida en que se aproxime a cero, se nota que también es muy bajo, tomando como referencia aquellos que estén igual o por debajo del 0,05, y nuevamente se encuentra la excepción en la variable 38; por lo tanto, esta variable debe salir de este grupo, desde el punto de vista cuantitativo, y desde el punto de vista cualitativo, esta competencia es más específica de la disciplina y menos general, por lo tanto, debe incluirse dentro de las competencias del saber. El grupo queda conformado de la siguiente manera:

1. Capacidad crítica.

2. Actitud crítica y creativa.

3. Rigor metodológico. El rigor implica humildad, capacidad de autocrítica y, a la vez, capacidad de crítica constructiva.

4. Pensamiento crítico.

5. Desarrollar el pensamiento crítico.

6. Análisis.

7. Autonomía.

\subsection{Competencias del ser.}

Se conformó seleccionando aquellas afirmaciones relacionadas con la ética (identificación y solución de problemas) y la comunicación (capacidad de argumentar y de comunicarse con los demás). Como en el análisis anterior, se recurrió a los estadísticos: frecuencias más altas, desviaciones más bajas y medias aritméticas más altas, quedando conformado el grupo de la forma como se aprecia en la tabla 3.2. 
Tabla 3. 2.

\section{Competencias del Ser}

\begin{tabular}{|c|c|c|c|c|}
\hline & & $\%$ & DS & MA \\
\hline 42. & $\begin{array}{l}\text { Habilidad para difundir sus } \\
\text { conocimientos en la comunidad } \\
\text { educativa y con el público en general. }\end{array}$ & 87 & 0.85 & 4.37 \\
\hline 41. & Conocer la eticidad investigativa. & 81 & 0.93 & 4.43 \\
\hline 26. & Competencia argumentativa. & 81 & 0.93 & 4.50 \\
\hline 6 & $\begin{array}{l}\text { Capacidad para aprender a generar } \\
\text { conocimiento pertinente. }\end{array}$ & 81 & 1.03 & 4.25 \\
\hline 43. & $\begin{array}{l}\text { Posibilidad de comprender los } \\
\text { problemas básicos en educación y } \\
\text { de difundirlos con sus pares y la } \\
\text { comunidad educativa. }\end{array}$ & 81 & 0.93 & 4.43 \\
\hline 50. & $\begin{array}{l}\text { Capacidad para actuar de manera } \\
\text { consecuente entre formación } \\
\text { y actuación. }\end{array}$ & 75 & 0.59 & 4.06 \\
\hline 37. & $\begin{array}{l}\text { La competencia para el Manejo de } \\
\text { alto nivel de la lengua materna, } \\
\text { especialmente en su tema. }\end{array}$ & 75 & 0.85 & 4.37 \\
\hline
\end{tabular}

DS: Desviación estándar.

MA: Media Aritmética

Al aplicarle el mismo análisis de correlación lineal simple y nivel de significación con los mismos parámetros, los números estadísticos demuestran que debe salir de este grupo la variable identificada con el número 37 (la competencia para el Manejo de alto nivel de la lengua materna, especialmente en su tema), la cual, en el cuadro anterior en el que se agruparon y se tuvieron en cuenta los parámetros estadísticos: frecuencia, desviación estándar y media aritmética, ocupó el último puesto, y en esta matriz presenta una baja correlación con las demás (por debajo del 0,54) y un nivel de significación mayor (por encima del 0,005). Las demás variables presentan una alta correlación entre sí y un nivel de significación aproximándose a cero (menos del 0,05); se destacan por su alta correlación y bajo nivel de significación las variables 6 y 43 (capacidad para aprender a generar conocimiento pertinente y la posibilidad de comprender los problemas básicos de la educación y de difundirlos con sus pares y la comunidad educativa respectivamente), las cuales fueron las únicas que se correlacionaron con la variable saliente.

El grupo de las competencias del ser queda entonces conformado así:

1. Habilidad para difundir sus conocimientos en la comunidad educativa y con el público en general.

2. Conocer la eticidad investigativa.

3. Competencia argumentativa.

4. Capacidad para aprender a generar conocimiento pertinente.

5. Posibilidad de comprender los problemas básicos en educación y de difundirlos con sus pares y la comunidad educativa. 


\subsection{Competencias del Saber.}

Se conformó un tercer grupo con las afirmaciones relacionadas con el dominio conceptual y metodológico, en el área educativa y en el contexto. A este grupo se le llamó competencias del Saber.

A continuación, en la tabla 3.3 se presentan las afirmaciones con sus respectivos valores de frecuencia (\%), desviación estándar (DS) y media aritmética (MA).

Tabla 3.3.

Competencias del saber

\begin{tabular}{|c|c|c|c|c|}
\hline & & $\%$ & DS & MA \\
\hline 10 & $\begin{array}{l}\text { Dominar conceptual y } \\
\text { metodológicamente los enfoques } \\
\text { de investigación en educación } \\
\text { y pedagogía. }\end{array}$ & 100 & 0.46 & 4.68 \\
\hline 18 & $\begin{array}{l}\text { La competencia investigativa para } \\
\text { transformar la realidad educativa. }\end{array}$ & 87 & 0.86 & 4.43 \\
\hline 33 & $\begin{array}{l}\text { La competencia para investigar, } \\
\text { innovar, liderar procesos. }\end{array}$ & 87 & 0.70 & 4.56 \\
\hline 57 & $\begin{array}{l}\text { Desarrollo de la } \\
\text { fundamentación teórica. }\end{array}$ & 87 & 0.70 & 4.50 \\
\hline 38 & Conocer a fondo la disciplina específica. & 85 & 0.75 & 4.25 \\
\hline 8 & $\begin{array}{l}\text { Capacidad para conocer los problemas } \\
\text { más significativos de los sistemas } \\
\text { educativos contemporáneos. }\end{array}$ & 82 & 0.78 & 4.62 \\
\hline 7 & $\begin{array}{l}\text { Capacidad para conocer en amplitud } \\
\text { y profundidad el campo de la educación } \\
\text { y la pedagogía. }\end{array}$ & 81 & 1.05 & 4.37 \\
\hline 22. & $\begin{array}{l}\text { Estar preparado para producir y llevar } \\
\text { a cabo investigaciones originales en su } \\
\text { campo, así como transmitirles su saber } \\
\text { en el campo académico, social y cultural. }\end{array}$ & 81 & 0.78 & 4.43 \\
\hline 34 & $\begin{array}{l}\text { Competencia para el manejo de la lectura } \\
\text { técnico-científica en lengua extranjera } \\
\text { (preferiblemente inglés). }\end{array}$ & 81 & 0.76 & 4.31 \\
\hline 37 & $\begin{array}{l}\text { La competencia para el manejo de alto } \\
\text { nivel de la lengua materna, especialmente } \\
\text { en su tema. }\end{array}$ & 75 & 0.85 & 4.37 \\
\hline 39 & $\begin{array}{l}\text { Fundamentos conceptuales de } \\
\text { investigación, en especial en el área. }\end{array}$ & 75 & 1.08 & 4.25 \\
\hline 45 & $\begin{array}{l}\text { Debe conocer muy bien el campo educativo, } \\
\text { al menos en lo que deben ser sus } \\
\text { enfoques centrales. }\end{array}$ & 75 & 1.14 & 4.25 \\
\hline 52 & $\begin{array}{l}\text { En la actualidad, debe tener claridad } \\
\text { que su quehacer investigativo debe } \\
\text { apuntar al desarrollo de las habilidades } \\
\text { para el siglo XXI. }\end{array}$ & 74.9 & 1.08 & 4.06 \\
\hline
\end{tabular}


Como se puede apreciar, existe un consenso sobre el dominio conceptual y metodológico de los enfoques en investigación y pedagogía que debe poseer un doctor en educación, y, a su vez, sobre la necesidad de ponerlos al servicio de la realidad educativa del país y de la región a través de la innovación y el liderazgo de procesos.

Desde un análisis cuantitativo y teniendo como parámetros una correlación lineal que va del 0.0 en adelante, hasta llegar a 1 y un nivel de significación menor de 0,05 aproximándose a cero, que es lo ideal, tenemos que las variables designadas con los números 8 (Capacidad para conocer los problemas más significativos de los sistemas educativos contemporáneos), 18 (La competencia investigativa para transformar la realidad educativa), 37 (La competencia para el manejo de alto nivel de la lengua materna, especialmente en su tema) y 57 (Desarrollo de la fundamentación teórica) quedan excluidas del grupo. En la forma como fueron redactadas, estas competencias van más enfocadas al hacer. De ahí que no sea de extrañar los resultados cuantitativos y la necesidad de que en educación se complementen estos dos análisis.

Lo mismo ocurre con la competencia 37 (Manejo de alto nivel de la lengua materna, especialmente en el tema). Su análisis presentaría una mayor correlación con las competencias del ser, en las cuales para su análisis se tuvo en cuenta lo relativo a la ética y la comunicación desde lo argumentativo, que con las competencias del saber.

En definitiva, el grupo de las competencias del Saber queda conformado de la siguiente manera:

1. Dominar conceptual y metodológicamente los enfoques de investigación en educación y pedagogía.

2. Conocer a fondo la disciplina específica.

3. Fundamentos conceptuales de investigación, en especial en el área.

4. Conocer en amplitud y profundidad el campo de la educación y la pedagogía.

5. La competencia para investigar, innovar, liderar procesos.

6. Estar preparado para producir y llevar a cabo investigaciones originales en su campo, así como transmitirles su saber en el campo académico, social y cultural.

7. Competencia para el Manejo de la lectura técnico-científica en lengua extranjera (preferiblemente inglés).

8. Debe conocer muy bien el campo educativo, al menos en lo que deben ser sus enfoques centrales.

9. En la actualidad, debe tener claridad que su quehacer investigativo debe apuntar al desarrollo de las habilidades propias del siglo XXI. 


\subsection{Competencias del Convivir.}

Un cuarto grupo se conformó con las afirmaciones de los expertos que se relacionaban con otros campos, otras disciplinas y el trabajo participativo. A este grupo se le denomina competencias del convivir que debe tener un doctor en educación. Realizando la primera fase del análisis estadístico, aplicado a los grupos anteriores, quedó conformado como se puede apreciar en la tabla 3. 4

Tabla 3. 4.

Competencias del Convivir

\begin{tabular}{|c|c|c|c|c|}
\hline & & $\%$ & DS & MA \\
\hline 11 & $\begin{array}{l}\text { Establecer relaciones de la educación } \\
\text { y la pedagogía con otros campos del } \\
\text { saber: sociología, economía, } \\
\text { antropología, psicología, entre otros. }\end{array}$ & 81 & 0.92 & 4.37 \\
\hline 40. & $\begin{array}{l}\text { Respeto a las diferentes } \\
\text { concepciones científicas. }\end{array}$ & 81 & 0.79 & 4.50 \\
\hline 21 & $\begin{array}{l}\text { Competencia en la comprensión de } \\
\text { las profundas articulaciones entre } \\
\text { la educación y lo político. }\end{array}$ & 80 & 0.78 & 4.43 \\
\hline 54 & Propiciar el trabajo colaborativo. & 75 & 0.94 & 4.18 \\
\hline
\end{tabular}

DS: Desviación estándar.

MA: Media Aritmética

A este selecto y pequeño grupo se le aplica la segunda fase del análisis estadístico que se ha venido aplicando (correlación Lineal simple), encontrando entre ellos unos altos niveles de correlación (aproximación a 1) y unos niveles de significación muy buenos (p se aproxima al cero). A excepción de las variables 11 (Establecer relaciones de la educación y la pedagogía con otros campos del saber: sociología, economía, antropología, psicología, entre otros) y 40 (Respeto a las diferentes concepciones científicas), que se salen de los parámetros anteriores, pero siguen teniendo vigencia porque un nivel $\mathrm{p}=0,07$ quiere decir que estamos trabajando con un nivel de confianza del 93\% y un margen de error del 7\% aproximadamente. En conclusión, este grupo continúa conformado por las afirmaciones iniciales sin ninguna modificación.

\subsection{Competencias del Hacer.}

El último grupo, lo conformaron las afirmaciones relacionadas con las competencias que requiere el doctor en educación para el desempeño profesional: competencias del Hacer, relacionadas con diseño, ejecución y dirección de propuestas y proyectos, con realización y exposición de publicaciones y reflexión sobre su quehacer, entre otras. Todo lo anterior dentro del ámbito educativo y con cobertura geográfica a todos los niveles. Con el análisis estadístico de frecuencia, desviación estándar y media aritmética quedó organizado el grupo de las competencias del hacer como se aprecia en la tabla 3. 5. 
Tabla 3.5.

\section{Competencias del Hacer}

$\%$

Competencia para elaborar propuestas de innovación curricular, del proceso de enseñanza-aprendizaje, lo cual incluye: métodos, medios y materiales, evaluación.

La competencia crítica para poner a prueba diferentes discursos y prácticas pedagógicas. comunicación de resultados.

Capacidad para redactar artículos científicos.

Competencia para construir teoría a partir de una reflexión permanente sobre la práctica, haciendo posible la unidad dialéctica praxis-reflexión acción - nuevos conocimientos.

Competencia para diseñar, implementar, ejecutar y evaluar proyectos de investigación del mayor nivel de su campo.

Competencia para saber leer la realidad educativa de su país, para formular problemas de investigación pertinentes a su contexto.

Competencia para esclarecer teorías pedagógicas, principios del aprendizaje en relación con la realidad socioeducativa.

15 Capacidad para dilucidar los grandes derroteros de los sistemas educativos.

55 Competencia para "saber leer adecuadamente" artículos cuantitativos y cualitativos, lo que se demuestra mediante la realización de análisis críticos.

Competencia para asesorar a diferentes instancias educacionales, tanto en las universidades como en el Ministerio de Educación; también, a empresas que realizan capacitación de su personal.

60 Capacidad para documentación (búsqueda experta).

Capacidad para exponer oralmente defender argumentos.

A

62 68

Actitudes hacia la investigación.

DS: Desviación estándar.

MA: Media Aritmética 
Una vez realizado el análisis de correlación lineal simple, salen las afirmaciones 9, 13, 59 y 61. El grupo de las competencias del doctor en educación, desde el hacer, se conformó de la siguiente forma:

1. Competencia para elaborar propuestas de innovación curricular del proceso de enseñanza-aprendizaje, lo cual incluye: métodos, medios y materiales, evaluación.

2. La competencia crítica para poner a prueba diferentes discursos y prácticas pedagógicas.

3. Competencia para construir teoría a partir de una reflexión permanente sobre la práctica, haciendo posible la unidad dialéctica praxis - reflexión - acción - nuevos conocimientos.

4. Competencia para saber leer la realidad educativa de su país, para formular problemas de investigación pertinentes a su contexto.

5. Competencia para esclarecer teorías pedagógicas, principios del aprendizaje en relación con la realidad socioeducativa.

6. Capacidad para dilucidar los grandes derroteros de los sistemas educativos.

7. Actitudes hacia la investigación.

8. Competencia para diseñar, implementar, ejecutar y evaluar proyectos de investigación del mayor nivel de su campo.

9. Capacidad analítica.

10. Capacidad propositiva.

11. Capacidad para realizar publicación/comunicación de resultados.

12. Capacidad para documentación (búsqueda experta).

13. Competencia para "saber leer adecuadamente" artículos cuantitativos y cualitativos, lo que se demuestra mediante la realización de análisis críticos.

\subsection{El Algoritmo de Johnson y las competencias del doctor en educación.}

El cuestionario utilizado para identificar las competencias del doctor, arrojó un coeficiente Alfa de Cronbach de 97,99\%. Esta confiabilidad se ve reflejada en las respuestas obtenidas por parte de los expertos, quienes, independientemente del país de procedencia, guardan una estrecha relación de similitud en cuanto a las respuestas. Esta afirmación se corrobora utilizando el estadístico de análisis multivariables, conocido como análisis de grupo, y dentro de este se escogió el estadístico del Algoritmo de Johnson con el método de encadenamiento completo. Lo primero que encontramos es la matriz conformada desde las distancias euclídeas y, después teniendo en cuenta 
esas distancias, se van conformando los diferentes grupos, se observa como unos quedan incluidos dentro de otros. Este apartado es expresado aquí numéricamente; y se aprecia en este mismo gráfico el lugar de procedencia del experto ( $\mathrm{PE}=$ Perú; $\mathrm{ESP}=$ España; VE= Venezuela; BOL= Bolivia; COL y CO = Colombia).

Análisis de grupos. Cluster Analysis

Tipo: Ascendente Algoritmo de Johnson Método de encadenamiento: Completo

\begin{tabular}{|c|c|c|c|c|c|c|c|c|c|c|c|c|c|c|c|c|}
\hline \multicolumn{17}{|c|}{ Matriz de distancias Euclídeas } \\
\hline 1 & 2 & 3 & 4 & 5 & 6 & 7 & 8 & 9 & 10 & 11 & 12 & 13 & 14 & 15 & 16 & 17 \\
\hline \multicolumn{17}{|c|}{ 1. PE 0,00} \\
\hline \multicolumn{17}{|c|}{ 2. PE $8,490,00$} \\
\hline \multicolumn{17}{|c|}{ 3. VE $9,8013,560,00$} \\
\hline \multicolumn{17}{|c|}{ 4. VE $7,353,7412,170,00$} \\
\hline \multicolumn{17}{|c|}{ 5. ESP $8,253,7413,194,000,00$} \\
\hline \multicolumn{17}{|c|}{ 6. ESP $8,899,9510,348,7710,150,00$} \\
\hline \multicolumn{17}{|c|}{ 7. ESP $8,545,5712,535,745,0010,300,00$} \\
\hline \multicolumn{17}{|c|}{ 8. COL 8,60 12,88 10,30 11,92 12,73 10,82 12,450,00 } \\
\hline \multicolumn{17}{|c|}{ 9. COL 7,87 3,16 13,04 4,00 2,45 9,75 5,20 12,08 0,00 } \\
\hline \multicolumn{17}{|c|}{ 10. COL $8,063,3213,003,612,6510,004,9013,003,000,00$} \\
\hline \multicolumn{17}{|c|}{ 11. COL 8,06 5,74 13,30 4,80 5,39 10,77 6,78 12,77 5,39 5,10 0,00 } \\
\hline \multicolumn{17}{|c|}{ 12. BOL $7,2810,449,339,4310,728,9410,109,759,9510,3910,390,00$} \\
\hline \multicolumn{17}{|c|}{ 13. COL $6,786,9310,776,006,328,067,2810,586,006,246,248,190,00$} \\
\hline \multicolumn{17}{|c|}{ 14. ESP $7,284,3612,924,583,8710,304,9012,213,613,465,8310,006,710,00$} \\
\hline \multicolumn{17}{|c|}{ 15. COL $7,684,3611,964,124,128,835,8312,123,614,246,168,946,564,690,00$} \\
\hline \multicolumn{17}{|c|}{ 16. PE $8,373,1613,113,462,4510,054,3613,042,831,735,0010,546,323,324,120,00$} \\
\hline 17. $\mathrm{CO} 9,54$ & & & & 3,75 & & & & & & & & & & & & \\
\hline
\end{tabular}

Proceso de formación de los grupos

\begin{tabular}{lll}
\hline Agrupación & Distancia Agrupamiento & Integrantes \\
\hline 1 & 1,73 & 1016 \\
2 & 2,45 & 59 \\
3 & 3,00 & 591016 \\
4 & 3,74 & 24 \\
5 & 3,87 & 59101614 \\
6 & 4,36 & 2415 \\
7 & 4,69 & 241559101614 \\
8 & 5,83 & 2415591016147 \\
9 & 6,24 & 1113 \\
10 & 7,28 & 112 \\
11 & 7,28 & 24155910161471113 \\
12 & 8,66 & 317 \\
13 & 8,94 & 1126 \\
14 & 10,30 & 3178 \\
15 & 10,77 & 112624155910161471113 \\
16 & 13,89 & 1126241559101614711133178
\end{tabular}

Grupo N. ${ }^{\circ} \quad$ Código de identificación de los integrantes

\begin{tabular}{lcl}
\hline 1 & 3 & 1126 \\
2 & 11 & 24155910161471113 \\
3 & 2 & 317 \\
4 & 1 & 8
\end{tabular}

Número de grupos retenidos: 4

Fuente: Elaboración propia autoras, con apoyo software Dyane 
Por último, se presentan los grupos que quedaron conformados. El grupo más significativo es el grupo No 2, que contiene 11 de las opiniones de los 17 expertos; se está frente a un grupo que contiene el 65\% de los expertos encuestados, todos de diferentes países, y sus opiniones coincidieron sobre las competencias que debe poseer un doctor en educación.

\section{CONCLUSIONES}

En la Región Andina, se encontró una tendencia a privilegiar la promoción y el desarrollo de las competencias del saber y el hacer. Es urgente, incluir dentro de las propuestas todo lo relacionado con las competencias del ser y del convivir, para asegurar la formación de doctores ciudadanos competentes que se insertan efectiva y positivamente en la comunidad académica nacional e internacional, capaces de interactuar globalmente dentro de un marco de convivencia ciudadana y asumir retos profesionales en el marco de una economía abierta, basada en el conocimiento con responsabilidad social.

Trabajar las competencias que conforman los básicos curriculares como un todo conjunto (metacompetencias, competencias del ser, saber, hacer y convivir), asegura a los doctores graduados en la región, desde cualquier universidad, su inserción en el espacio mundial; será un doctor formado en la región con las competencias de cualquier doctor formado en el extranjero y competente para desempeñarse en el ámbito educativo, a cualquier nivel, local, regional, nacional e internacional.

Para este trabajo se realizaron análisis por cada una de las dimensiones, para de esta manera facilitar los consensos por grupos de competencias, pero los lectores se dan cuenta como estas competencias se complementan entre sí y se necesitan mutuamente, tanto dentro del grupo como intergrupos de competencias; por ejemplo, la capacidad crítica, requiere de un desarrollo del pensamiento crítico y a su vez, este, requiere de competencias como capacidad de búsquedas expertas, dominios conceptuales, teóricos y metodológicos, en el área educativa, de manera que pueda producir nuevos conocimientos, socializarlos de forma oral y escrita para validarlos y reflexionarlos con su comunidad académica nacional e internacional.

Todo lo anterior lleva implícitos cambios en las formas y criterios de evaluación del doctor, cuya tendencia ha sido una evaluación final, desde la presentación y sustentación de la tesis doctoral. Es necesario evaluarlo desde una mirada integral (ser, convivir, saber y hacer) tanto, durante el proceso de formación, como en un trabajo final, y las tesis girar alrededor de problemas complejos con relevancia científica y trascendencia social.

Queda demostrado como las competencias trabajadas como básicos curriculares, se constituyen en estrategias de armonización e integración de regiones formando doctores que respondan a las necesidades propias de sus países y regiones como a las necesidades del mundo globalizado en el que actualmente nos estamos desenvolviendo 


\section{REFERENCIAS BIBLIOGRÁFICAS}

Cronbach, L (1951). Coefficient alpha and the internal structure of test. psychometrika, 16 (september), 297-334.

Dalkey, N (1969). The delphi method: an experimental study of group opinion. Santa Mónica, Ca: Rand corp.

Delbecq, A., Van de Ven, H., Gustafson, H. (1975). Group techniques for program planning . Glenview, Il: Scott-Foresman.

Delors, J (1996). La educación encierra un tesoro. Informe a la UNESCO de la comisión internacional sobre la educación para el siglo XXI. Madrid: Santillana.

Delors, J (1996). La educación encierra un tesoro. Informe a la UNESCO de la comisión internacional

Escobar-Espinoza, A., \& Angulo-Pico, G. (2015). Panorama Economico in the context of the international visibility of scientific journals. Panorama Económico, 23, 11-13. DOI: https://doi.org/10.32997/2463-0470-vol.23-num.1-2015-1733

García, J (2000). Orientaciones para el estudio de la educación comparada. Madrid: UNED.

Gonezi, A (1996) Instrumentación de la educación basada en competencias, perspectivas de la teoría y la práctica en Australia. Limaza.

Gutnar, G (2001). $3^{\circ}$ Congreso Competencias, educación y formación profesional. Interpretación de los componentes de la formación que intervienen en la actitud investigativa de los estudiantes universitarios. Barranquilla. Colombia.

Linstone, A., and Turoff, M., ed.,(1975). The deiphl method: technique and aplications. Massachusetts.

Magendzo, K. (1.996). Curriculum, Educación para la democracia en la modernidad. Programa interdisciplinario de investigación en educación. Bogotá: Ediciones Antropos.

Martínez, A., Calderón, J., García, M., Vásquez, M., Rodríguez, R., (2005). Perfil de competencias del tutor de postgrado. México: Universidad Autónoma.

Moreno, B.M (2005). Los retos del postgrado en la educación. Recuperado el día 22 de Abril 2007 del sitio http://educacion.jalisco.gob.mx/dependen/posgrados/Posgrado/html/

Navio, A (2001) Las competencias del formador de formación continua. Análisis desde los programas de formación de formadores. Tesis doctoral. Universidad Autónoma de Barcelona. Bellaterra.

Passos-Simancas, E., \& Arias-Aragonés, F. (2016). El capital humano como factor de competitividad en la industria hotelera y turística en Cartagena. Panorama Económico, 24, 269 - 282. DOI: https://doi.org/10.32997/2463-0470-vol.24-num.0-2016-1561

Sandoval, A (1996).Módulos de investigación social. Bogotá: ICFES.

Santesmases, M (1997). Diseño y análisis de encuestas en investigación social y de mercados. Madrid: Ediciones Pirámide.

Tuning Educational Structures In Europa (2003). Informe final. Fase uno. Editada por González, J. Universidad Deusto, y Universidad de Groningen.

Vergara-De la Ossa, R., Londoño-Vega, C., Pérez-Benítez, N., \& Torres-Castellar, R. (2015). La adopción de las Normas Internacionales de Información Financiera en Colombia. Panorama Económico, 23, 119-132. DOI: https://doi.org/10.32997/2463-0470-vol.23num.1-2015-1382 